\title{
MODIFICATIONS OF FOURIER TRANSFORMS ${ }^{1}$
}

\author{
WALTER RUDIN
}

A classical theorem of Wiener [3, p. 108], [1, p. 118] shows that the presence or absence of the discrete component of a measure is accurately and quantitatively reflected in the asymptotic behavior of its Fourier transform.

On the other hand, it must be obvious to anyone who has worked in this area that there cannot exist any good general criterion which would enable us to deduce from the asymptotic behavior of the Fourier transform of a continuous measure $\mu$ whether $\mu$ does or does not have a singular component. The present note contributes further evidence in this direction by showing that in many groups (including the real line and the integers) there are relatively small sets on which the Fourier transform of any absolutely continuous measure can be so modified that it becomes the transform of a singular measure.

Let $\Gamma$ be the dual of a locally compact abelian group $G ; L^{1}(G)$ and $M(G)$ denote the spaces of all Haar-integrable functions on $G$ and of all complex Borel measures on $G$, respectively, and we identify $L^{1}(G)$ with the absolutely continuous members of $M(G)$. The Fourier transform of $\mu \in M(G)$ is defined to be

$$
\hat{\mu}(\gamma)=\int_{G}(-x, \gamma) d \mu(x) \quad(\gamma \in \Gamma),
$$

where $(x, \gamma)$ is the value of the character $\gamma$ at the point $x \in G$. If $f \in L^{1}(G), \hat{f}(\gamma)$ is defined by (1) with $f(x) d x$ in place of $d \mu(x)$, where $d x$ denotes the Haar measure of $G$. All groups will be written additively. Notation and terminology are exactly as in [1].

TheOREM. Suppose $\Omega$ is a subset of $\Gamma$ with the following property: to each compact set $K \subset \Gamma$ there corresponds an infinite discrete closed subgroup $\Lambda$ of $\Gamma$ such that

$$
(K-K) \cap \Lambda=\{0\}
$$

and such that $K+\lambda \subset \Omega$ for every $\lambda \in \Lambda$, except for $\lambda=0$.

Received by the editors May 9, 1967.

${ }^{1}$ Research sponsored by the Air Force Office of Scientific Research, Office of Aerospace Research, United States Air Force, under AFOSR Grant No. 1160-66, and by the Wisconsin Alumni Research Foundation. 
Then there corresponds to every $f \in L^{1}(G)$ a $\mu \in M(G)$, singular with respect to the Haar measure of $G$, such that

$$
\hat{\mu}(\gamma)=\hat{f}(\gamma) \quad \text { for all } \gamma \text { outside } \Omega .
$$

Before we prove the Theorem we give some examples of situations in which it can be applied.

EXAMPLE 1. $G=\Gamma=R^{1}$, the real line. Let $p_{1}<p_{2}<p_{3}<\cdots$ be positive integers, let $\Omega_{s}$ be the union of the intervals $\left[n p_{s}-s, n p_{s}+s\right]$, for $n= \pm 1, \pm 2, \pm 3, \cdots$ (note that $n=0$ is omitted!) and put $\Omega$ $=\Omega_{1} \cup \Omega_{2} \cup \Omega_{3} \cup \ldots$. If $K$ is compact, then $K \subset[-s, s]$ for some $s$, and we can take the infinite cyclic group generated by $p_{s}$ as our $\Lambda$.

If $\epsilon>0$ we can choose $\left\{p_{s}\right\}$ so that $\sum 2 s / p_{s}<\epsilon$. Then $\Omega$ will be small in the sense that

$$
m(\Omega \cap[-t, t])<2 t \epsilon
$$

for every positive number $t$. Here $m$ denotes Lebesgue measure, of course; (4) implies that the upper density of $\Omega$ is at most $\epsilon$.

The same construction can of course be carried out if $\Gamma=Z$, the additive group of the integers.

Example 2. $G=\Gamma=R^{2}$, the plane. Define

$$
\Omega=\{(s, t):|s|>10,|t|<\log \log |s|\} .
$$

Given a compact $K \subset R^{2}$ let $\Lambda$ be the group generated by $\left(s_{K}, 0\right)$ where $s_{K}$ is suitably large. This $\Omega$ has density 0 , in an obvious sense.

ExAmple 3. $G=T^{N}$, the $N$-dimensional torus, $\Gamma=Z^{N}$, the additive group of all $N$-tuples $\left(n_{1}, \cdots, n_{N}\right)$ with $n_{i} \in Z$. Let $P$ be the set of all $\gamma \in Z^{N}$ for which all $n_{i}$ are nonnegative ( $P$ is the positive quadrant, octant, etc., in $Z^{N}$ ), let $-P$ be the set of all $\gamma$ such that $-\gamma \in P$, and put $\Omega=P \cup(-P)$. The density of $\Omega$ is $2^{1-N}$.

If $K$ is compact in $Z^{N}$ then $K$ is finite, and $\Lambda$ can be taken to be the cyclic group generated by a suitable multiple of $(1,1, \cdots, 1)$.

A case of this example occurred in [2]. Our theorem has relevance to the theory of several complex variables for the following reason: $T^{N}$ can be embedded in $C^{N}$, the space of $N$ complex variables, so as to be the distinguished boundary of the unit polydisc $U^{N}$. If the given function $f$ on $T^{N}$ is real, $\mu$ can also be taken real (as the proof will show), and since $\hat{\mu}=\hat{f}$ except on $\Omega$, the Poisson integral of $f-d \mu$ will define a function $u$ in $U^{N}$ which is the real part of a holomorphic function and whose radial limits are equal to $f$ a.e. on $T^{N}$, since $\mu$ is singular. (See [3, Chapter 17] for Poisson integrals in several variables.)

EXAmple 4. Let $G$ be the infinite-dimensional torus $T^{\omega}$ whose dual $Z^{\infty}$ consists of all integer sequences $\left\{n_{1}, n_{2}, n_{3}, \cdots\right\}$ with only finitely 
many $n_{i}$ different from 0 . As in Example 3 , let $\Omega$ consist of all $\gamma \in Z^{\infty}$ which have all $n_{i} \geqq 0$, plus those which have all $n_{i} \leqq 0$.

If $K$ is compact in $Z^{\infty}$ then $K$ is finite, and there is an integer $r$ such that every $\gamma \in K$ has $n_{i}=0$ if $i>r$ and has $\left|n_{i}\right|<r$ if $1 \leqq i \leqq r$. Choose $\gamma_{K} \in Z^{\infty}$ so that $n_{1}=\cdots=n_{r}=2 r, n_{i}=0$ for $i>r$, and associate the group $\Lambda$ generated by $\gamma_{K}$ to $K$.

We now turn to the proof of the theorem.

Lemma 1. If $f \in L^{1}(G)$ and $\hat{f}$ has compact support then there exists $g \in L^{1}(G)$ and $u \in C_{c}(G)$ such that

$$
f=f * g * u \text {. }
$$

Here $C_{c}(G)$ is the space of all continuous functions on $G$, with compact support, and $*$ denotes convolution.

Proof. Let $K$ be the support of $\hat{f}$. By taking the support of $u$ in a small enough neighborhood of 0 we can find $u \in C_{c}(G)$ such that $|\hat{u}(\gamma)| \geqq 1$ for all $\gamma \in K$. There exists $v \in L^{1}(G)$ such that $\hat{v}(\gamma)=1$ on $K$ and $\hat{v}(\gamma)=0$ wherever $|\hat{u}(\gamma)| \leqq \frac{1}{2}[1$, Theorem 2.6.1]. It follows that there is a continuous function $\phi$ on $\Gamma$ such that $\phi \hat{a}=\hat{v}$ and such that $\phi(\gamma)=0$ wherever $\hat{v}(\gamma)=0$.

Suppose $\gamma_{0} \in \Gamma$ is such that $\phi$ does not vanish identically in any neighborhood of $\gamma_{0}$. Then $u\left(\gamma_{0}\right) \neq 0$. Put $c=u\left(\gamma_{0}\right)$. By [1, Theorem 2.6.5] there exists $h \in L^{1}(G)$ with $\|h\|_{1}<|c|$, such that $a(\gamma)+\hat{h}(\gamma)=c$ in a neighborhood $U$ of $\gamma_{0}$. The series

$$
\hat{v} \cdot \sum_{n=0}^{\infty} c^{-n-1} \hat{h}^{n}
$$

converges in the norm of $A(\Gamma)$ (the space of all Fourier transforms of members of $L^{1}(G)$ ), so its sum is $\hat{w}$ for some $w \in L^{1}(G)$, and one computes easily that $\hat{w}(\gamma)=\phi(\gamma)$ in $U$.

So $\phi$ belongs to $A(\Gamma)$ locally, and it follows from [1, Theorem 6.2.6] that $\phi=\hat{g}$ for some $g \in L^{1}(G)$.

Since $\hat{v}(\gamma)=1$ on $K$, we have $\hat{f}=\hat{f} \hat{v}=\hat{f} \hat{g} \hat{u}$, and this is equivalent to (6).

Lemma 2. Suppose $f \in L^{1}(G), \hat{f}$ has compact support $K$, and $\Lambda$ is an infinite discrete closed subgroup of $\Gamma$ such that (2) holds. Then there exists $\mu \in M(G)$, singular with respect to the Haar measure of $G$, such that

(i) $\hat{u}(\gamma)=\hat{f}(\gamma)$ for $\gamma \in K$,

(ii) the support of $\hat{\mu}$ is $K+\Lambda$,

(iii) $\|\mu\| \leqq\|f\|_{1}$.

Here $\|\mu\|$ denotes the total variation of $\mu$. 
Proof. Let $H$ be the annihilator of $\Lambda$ [1, p. 35], i.e., $H$ is the set of all $x \in G$ at which $(x, \lambda)=1$ for every $\lambda \in \Lambda$. Clearly $H$ is a closed subgroup of $G$, and since $\Lambda$ is discrete, the quotient group $G / H$ is compact. Let $d x, d s, d \xi$ denote the Haar measures of $G, H, G / H$. We normalize $d \xi$ so that its total mass is 1 (since $G / H$ is compact) and we can then adjust $d s$ so that the formula

$$
\int_{G} \phi(x) d x=\int_{G / H} d \xi \int_{H} \phi(x+s) d s
$$

holds for every $\phi \in C_{c}(G)[1$, p. 54]. Here and later $\xi=\xi(x)$ is the coset of $H$ which contains $x$.

Since $\hat{f}$ has compact support, the inversion theorem shows that $f$ coincides almost everywhere with a continuous function. So we may assume that $f$ is continuous, and we define

$$
\beta(\xi)=\int_{H}|f(x+s)| d s \quad(\xi \in G / H) .
$$

It follows from (8) that $\beta(\xi)<\infty$ for almost all $\xi$, but Lemma 1 implies that $\beta$ is actually a continuous function: Lemma 1 gives

$$
\begin{aligned}
\left|f\left(x^{\prime}+s\right)-f\left(x^{\prime \prime}+s\right)\right| \leqq & \int_{G}|(f * g)(x)| \\
& \cdot\left|u\left(x^{\prime}+s-x\right)-u\left(x^{\prime \prime}+s-x\right)\right| d x
\end{aligned}
$$

and since $u \in C_{c}(G)$ there corresponds to each $\epsilon>0$ a neighborhood $V$ of 0 in $G$ such that $x^{\prime}-x^{\prime \prime} \in V$ implies

$$
\int_{H}\left|f\left(x^{\prime}+s\right)-f\left(x^{\prime \prime}+s\right)\right| d s<\epsilon .
$$

The continuity of $\beta$ now follows from (10).

Now fix $\xi_{0} \in G / H$ so that $\beta\left(\xi_{0}\right) \leqq \beta(\xi)$ for all $\xi \in G / H$, fix $x_{0} \in \xi_{0}$, and define $\tau \in M(G)$ by

$$
d \tau=f\left(x_{0}+s\right) d s .
$$

Then $\tau$ is concentrated on $H$,

$$
\|\tau\|=\beta\left(\xi_{0}\right) \leqq \int_{G / H} \beta(\xi) d \xi=\|f\|_{1}
$$

and, for any $\gamma \in \Gamma$, 


$$
\begin{aligned}
\hat{\tau}(\gamma) & =\int_{H}(-s, \gamma) f\left(x_{0}+s\right) d s \\
& =\left(x_{0}, \gamma\right) \int_{H}\left(-x_{0}-s, \gamma\right) f\left(x_{0}+s\right) d s=\left(x_{0}, \gamma\right) F_{\gamma}\left(\xi_{0}\right)
\end{aligned}
$$

where

$$
F_{\gamma}(\xi)=\int_{H}(-x-s, \gamma) f(x+s) d s \quad(\xi \in G / B) .
$$

If we multiply $f$ by a character, the analogue of (10) still holds, and we see that each $F_{\gamma}$ is a continuous function on $G / H$.

We wish to determine $F_{\gamma}$ by computing its Fourier coefficients. The characters of $G / H$ are the members of $\Lambda$, but each $\lambda \in \Lambda$ is also a character of $G$ which is constant on the cosets of $H$. Hence we can identify $(x, \lambda)$ and $(\xi, \lambda)$. With this in mind we obtain

$$
\begin{aligned}
\hat{F}_{\gamma}(\lambda) & =\int_{G / H}(-\xi, \lambda) F_{\gamma}(\xi) d \xi \\
& =\int_{G / H} d \xi \int_{H}(-x-s, \gamma+\lambda) f(x+s) d s=\hat{f}(\gamma+\lambda)
\end{aligned}
$$

for any $\gamma \in \Gamma, \lambda \in \Lambda$.

If $\gamma$ is not in $K+\Lambda,(15)$ shows that $\hat{F}_{\gamma}(\lambda)=0$ for every $\lambda \in \Lambda$; hence $F_{\gamma}(\xi)=0$ for all $\xi$, and (13) gives $\hat{\tau}(\gamma)=0$. So the support of $\hat{\tau}$ is in $K+\Lambda$.

If $\gamma \in K$, (2) implies that $\gamma+\lambda \in K$ if and only if $\lambda=0$, so (15) shows that $\hat{F}_{\gamma}(\lambda)=0$ if $\lambda \neq 0$ and that $\hat{F}_{\gamma}(0)=\hat{f}(\gamma)$. Hence $F_{\gamma}(\xi)=\hat{f}(\gamma)$ for all $\xi$, and (13) gives

$$
\hat{\tau}(\gamma)=\left(x_{0}, \gamma\right) \hat{f}(\gamma) \quad(\gamma \in K) .
$$

Since $\tau$ is concentrated on $H, \hat{\tau}(\gamma+\lambda)=\hat{\tau}(\gamma)$ for all $\lambda \in \Lambda$; hence the support of $\hat{\tau}$ is exactly $K+\Lambda$.

Next we claim that $\tau$ is singular. To prove this it is enough to show that every compact set $E \subset H$ has Haar measure 0 in $G$. If $h$ is the characteristic function of $E$, then $\hat{h}(\lambda)=m(E)$ for every $\lambda \in \Lambda$, and since $\Lambda$ lies in no compact subset of $\Gamma$, the fact that $\hat{h}$ vanishes at infinity implies $m(E)=0$.

Finally, define $\mu \in M(G)$ by: $\hat{\mu}(\gamma)=\left(-x_{0}, \gamma\right) \hat{\tau}(\gamma)$ for all $\gamma \in \Gamma$. This clearly satisfies all requirements. 
Proof of the Theorem. The functions whose Fourier transforms have compact support are dense in $L^{1}(G)$. So if $f \in L^{1}(G)$ we can represent $f$ in the form $f=\sum f_{i}$ where each $\hat{f}_{i}$ has compact support $K_{i} \subset \Gamma$ and where $\sum\left\|f_{i}\right\|_{1}<\infty$. Apply Lemma 2 to each $f_{i}$, obtaining singular measures $\mu_{1}$ such that $\hat{\mu}_{i}(\gamma)=\hat{f}_{i}(\gamma)$ outside $\Omega$ (by (i) and (ii) of Lemma 2) and such that $\left\|\mu_{i}\right\| \leqq\left\|f_{i}\right\|_{1}$. The series $\sum \mu_{i}$ then converges in the total variation norm to a measure $\mu$ which is therefore also singular, and if $\gamma$ is not in $\Omega$ we have

$$
\hat{\mu}(\gamma)=\sum \hat{\mu}_{i}(\gamma)=\sum \hat{f}_{i}(\gamma)=\hat{f}(\gamma) .
$$

ADDED IN PROOF. Further results along these lines have appeared in Bull. Amer. Math. Soc. 74 (1968), 526-528.

\section{REFERENCES}

1. Walter Rudin, Fourier analysis on groups, Interscience, New York, 1962.

2. - Inner functions in polydiscs, Bull. Amer. Math. Soc. 73 (1967), 369-372.

3. Antoni Zygmund, Trigonometric series, 2nd ed., Cambridge Univ. Press, New York, 1959.

UNIVERSITY OF WISCONSIN AND

University of California, San Diego 